\title{
Diagnostic Value of Diffusion-Weighted MRI for Tumor Characterization, Differentiation and Monitoring in Pediatric Patients with Neuroblastic Tumors
}

\section{Diagnostischer Stellenwert der diffusionsgewichteten MRT zur Tumorcharakterisierung, Tumordifferenzierung und zur Verlaufskontrolle bei pädiatrischen Patienten mit neuroblastischen Tumoren}

Authors

Henning Neubauer ${ }^{1,4}$, Mengxia Li $^{2}$, Verena Rabea Müller ${ }^{3}$, Thomas Pabst ${ }^{4}$, Meinrad Beer ${ }^{1}$

Affiliations

1 Department of Diagnostic and Interventional Radiology, University Hospital Ulm, Germany

2 Department of Radiation Oncology, University Hospital of Würzburg, Germany

3 Department of Paediatrics, University Hospital of Würzburg, Germany

4 Department of Diagnostic and Interventional Radiology, University Hospital of Würzburg, Germany

Key words

neuroblastoma, ganglioneuroblastoma, ganglioneuroma, therapy response, MR imaging, MR diffusion/perfusion

received 03.10 .2016

accepted 22.03.2017

Bibliography

DOI https://doi.org/10.1055/s-0043-108993

Published online: 16.5.2017 | Fortschr Röntgenstr 2017; 189: 640-650 @ Georg Thieme Verlag KG, Stuttgart · New York, ISSN 1438-9029

Correspondence

Dr. Henning Neubauer

Department of Diagnostic and Interventional Radiology,

University Hospital of Ulm, Albert-Schweitzer-Allee 23,

$89081 \mathrm{Ulm}$

Tel.: ++ 49/731/50061001

Fax: $++49 / 731 / 50061002$

inu75@web.de

\section{ZUSAMMENFASSUNG}

Ziel Untersucht wurde die diagnostische Aussagekraft der diffusionsgewichteten MRT (DWI) für die Tumorcharakterisierung, Tumordifferenzierung und Verlaufskontrolle bei Kindern mit extrakraniellen neuroblastischen Tumoren.

Material und Methoden Alle 29 Patienten (14 Mädchen, medianes Alter 3 Jahre) mit Neuroblastom (NB, $n=19)$, Ganglioneuroblastom (GNB, $n=4$ ) und Ganglioneurom (GN, $n=6$ ) und mindestens einer DWI-Studie in domo seit 2005 wurden retrospektiv analysiert. Zwei unabhängige geblindete Untersucher bestimmten die ADC-Werte (Einheit: $10-3 \mathrm{~mm}^{2} / \mathrm{s}$ ) und Signalintensitätsquotienten (SIR) für Primärtumor und, falls zutreffend, für Tumor nach Chemotherapie, Metastasen und Tumorrezidiv.

Ergebnisse Initial betrugen der ADC-Wert 0,90 $\pm 0,23$ für NB/GNB und 1,70 $\pm 0,36$ für GN ohne Überlappung beider Entitäten bei beiden Untersuchern, 0,67 $\pm 0,14$ für Metastasen und 0,72 $\pm 0,18$ für Rezidive. Unter Chemotherapie stieg der ADC auf 1,54 $\pm 0,33$ bei NB/GNB und 1,23 $\pm 0,27$ bei Metastasen $(p<0,05)$. Die medianen SIR der Tumorläsionen vs. Leber, vs. Muskel und vs. benachbartes Gewebe waren signifikant höher in der DWI $(\min . . . \max 2,4 \ldots 9,9)$ als in ce-T1w $(1,0 \ldots 1,8$, alle $p<0,05)$. Der Variationskoeffizient (CV) lag $\leq 8,0 \%$ für ADC und $\leq 16,4 \%$ für SIR.

Schlussfolgerung DWI unterscheidet anhand des ADC zuverlässig zwischen NB/GNB und GN und liefert plausible quantitative Daten zum Therapieansprechen. Die Sichtbarkeit von Tumorherden, gemessen als SIR, ist in der DWI deutlich besser als in der ce-T1w. DWI als nicht-invasive und weithin verfügbare Bildgebungstechnik ohne Strahlenexposition sollte integraler Bestandteil jeder MR-Bildgebung bei neuroblastischen Tumoren sein und sollte in multizentrischen Studien prospektiv evaluiert werden.

\section{Kernaussagen}

- DWI differenziert anhand des ADC-Werts zuverlässig zwischen Neuroblastom/Ganglioneuroblastom und Ganglioneurom.

- Die DWI liefert plausible quantitative Daten zum Therapieansprechen.

- Die Erkennbarkeit von Tumorherden ist höher mit DWI als mit ce-T1w.

- DWI sollte als Standardtechnik für die MR-Bildgebung neuroblastischer Tumoren betrachtet werden. 


\section{ABSTRACT}

Purpose We explored the diagnostic value of diffusionweighted MRI (DWI) for tumor characterization, differentiation and therapy monitoring in pediatric patients with extracranial neuroblastic tumors.

Materials and Methods All 29 patients (14 girls, median age: 3 years) with neuroblastoma (NB, $n=19)$, ganglioneuroblastoma ( $G N B, n=4)$ and ganglioneuroma ( $G N, n=6$ ) who had had at least one in-house DWI examination since 2005 were identified and retrospectively analyzed. Two independent blinded readers measured ADC values (unit: $10-3 \mathrm{~mm}^{2} / \mathrm{s}$ ) and signal intensity ratios (SIRs) of the primary tumor and, if applicable, of the tumor after chemotherapy, metastases and tumor relapse.

Results The pre-treatment ADC was $0.90 \pm 0.23$ in NB/GNB and $1.70 \pm 0.36$ in $G N$ without overlap between the two entities for both readers, $0.67 \pm 0.14$ in metastases and $0.72 \pm 0.18$ in tumor relapse. With chemotherapy, mean ADC increased to $1.54 \pm 0.33$ in NB/GNB and to $1.23 \pm 0.27$ in metastases $(p<0.05)$. The median SIRs of various tumor lesions vs. liver, vs. muscle tissue and vs. adjacent tissue were significantly higher on DWI (range: 2.4 -9.9) than on ce-T1w (range: $1.0-1.8$, all $p<0.05$ ). The coefficient of variation (CV) was $\leq 8.0 \%$ for ADC and $\leq 16.4 \%$ for signal intensity data.
Conclusion Based on mean ADC, DWI distinguishes between NB/GNB and GN with high certainty and provides plausible quantitative data on tumor response to therapy. Lesion conspicuity, as measured by SIR, is superior on DWI, compared to ce-T1w. DWI as a noninvasive, radiation-free and widely available imaging technique should be an integral part of MR imaging for neuroblastic tumors and should undergo prospective evaluation in multicenter studies.

\section{Key Points}

- DWI reliably distinguishes neuroblastoma/ganglioneuroblastoma from ganglioneuroma, based on the mean ADC.

- DWI provides plausible quantitative data on tumor response to chemotherapy.

- DWI offers highly superior lesion conspicuity compared to contrast-enhanced T1w imaging.

- DWI should be considered a standard for imaging neuroblastic tumors.

\section{Citation Format}

- Neubauer H, Li M, Müller VR et al. Diagnostic Value of Diffusion-Weighted MRI for Tumor Characterization, Differentiation and Monitoring in Pediatric Patients with Neuroblastic Tumors. Fortschr Röntgenstr 2017; 189: 640-650

\section{Introduction}

Neuroblastic tumors comprise a broad spectrum of neoplasia with highly diverse biological behavior, ranging from undifferentiated, highly malignant neuroblastoma (NB) to mature, usually benign ganglioneuroma (GN) [1]. NB is the most common extracranial malignant solid tumor in pediatric patients, accounting for about $8 \%$ of all childhood malignancies [2, 3]. Based on standard imaging techniques, including radiography, ultrasound, CT and conventional MRI scans, differentiation between NB, ganglioneuroblastoma (GNB) and $\mathrm{GN}$ is not possible with diagnostic confidence $[4,5]$. Therefore, the role of diagnostic imaging in the clinical workup of patients with neuroblastic tumors is presently limited to detecting, or excluding, metastatic disease and local complications, such as infiltration of organs and vascular structures $[6,7]$, while over-all risk stratification relies on clinical, histopathological and genetic criteria [8]. Diffusion-weighted MRI (DWI) has, with some success, been introduced as an adjunct to pediatric oncological MR imaging protocols [9]. Preliminary results suggest that, based on apparent diffusion coefficient (ADC) values, DWI can indeed distinguish between NB, GNB and GN with some confidence $[10,11]$. Recent reports confirm these findings [12] and propose diagnostic value for detecting metastases [13] and for monitoring therapy response [14].

We undertook this study to test our assumptions of a substantial diagnostic value of DWI in comparison to standard contrastenhanced MRI for tumor differentiation, therapy response and imaging of metastases in a comparably large cohort of children with extracranial neuroblastic tumors from a single center.

\section{Materials and Methods}

\section{Patients}

Based on PACS queries, tumor board documents and medical history reports, we retrospectively identified all patients with extracranial neuroblastic tumors who underwent imaging and treatment at our institution between January 1, 2005, and September 5, 2016 ( $\triangleright$ Fig. 1). Screening all MRI examinations of these patients for DWI scans, we eventually enrolled all 29 patients (14 girls, median age at primary diagnosis: 3 years, range: 2 months to 19 years) who had at least one MRI study with DWI at our institution. All eligible sets of imaging data containing both standard and diffusion-weighted MRI were identified and assigned to the following categories: (1) initial imaging/before therapy $n=18$ patients with primary tumor location cervical $n=3$, adrenal $n=11$, paravertebral-retroperitoneal $n=2$ and intrapelvic $n=2$; (2) evaluating therapy response $n=15$ patients; (3) synchronous metastasis before therapy $n=4$ patients with 8 lesions; (4) synchronous metastases under treatment $n=6$ patients with 10 lesions; (5) patients with relapse/recurrence $n=5$ patients with 9 lesions. If more than one examination per patient was available in one category, only the first eligible MRI scan was used for analysis.

Treatment plans were determined by interdisciplinary tumor conferences in cooperation with the national study coordination office. NB/GNB patients had initial tumor biopsy and were then referred for further treatment. Chemotherapeutic treatment at our institution is based on the NB-2004 trial protocol and its later 


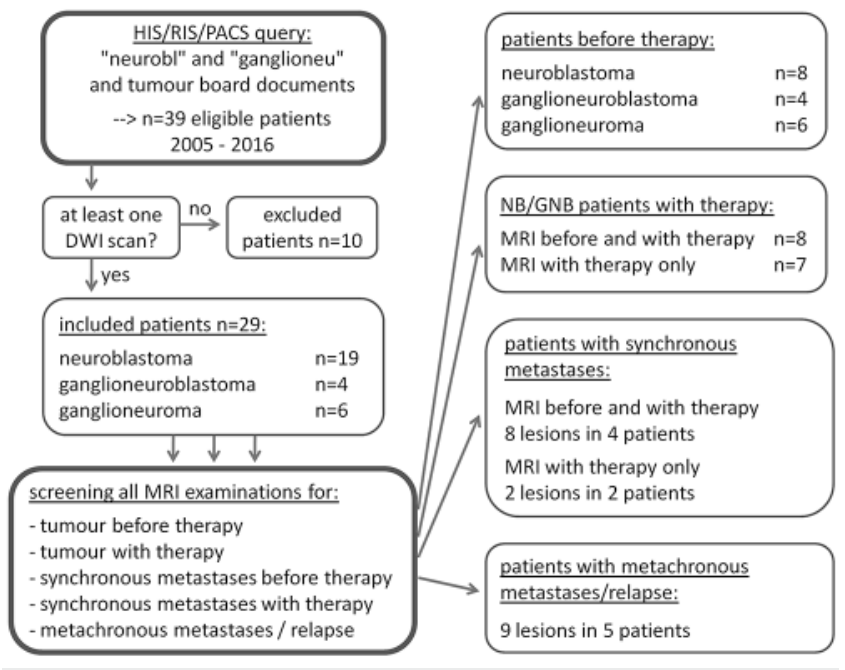

- Fig. 1 Flowchart showing the process of patient selection and the patient groups for data analysis.

- Abb. 1 Flow chart zum Modus der Patientenselektion und zur Gruppeneinteilung für die Datenanalyse.

modifications. GN patients underwent tumor resection. Histopathological correlation was available in all patients at first diagnosis, confirming NB in 19 (stage $2 n=3$, stage $3 n=7$, stage $4 n=7$ and stage $4 \mathrm{~s} n=2$ [15]), GNB in 4 and $G N$ in 6 patients. MYCN gene amplification status was available in 18 of 19 NB patients (10 MYCN-positive, 8 MYCN-negative) and 3 of 4 GNB patients (all 3 MYCN-negative). Information on MYCN gene amplification was not available in one GNB patient diagnosed in 2005 and in one NB patient from abroad who was transferred to our institution for treatment of tumor recurrence. Histological correlation was available in three patients with metastases and/or tumor relapse. The remaining lesions were retrospectively verified as malignant based on the following criteria: typical characteristics on MRI suggestive of tumor and new or growing lesion compared to previous imaging and/or significant regression in response to treatment and/or progression in spite of treatment.

All study work was conducted in accordance with the Helsinki Declaration. Informed written consent was obtained from the patients and/or the legal guardians for all diagnostic procedures. A waiver was granted by the Institutional Review Board for the retrospective analysis of imaging data.

\section{MRI examination}

All patients underwent clinical routine MRI at 1.5 Tesla (Magnetom Symphony $n=19$, Magnetom Avanto $n=3$, Magnetom Aera $\mathrm{n}=32$ ) and 3 Tesla (Magnetom Trio $n=2$, two scans of cranial osseous metastases) in supine position with an intravenous line in place. A head-and-neck coil was used for cervical scans and phased-array body coils for thoracic-abdominal examinations. Standard pre-contrast sequences were scanned as needed for clinical diagnostic workup (e.g. coronal T2w TIRM, transverse T2w HASTE and/or T2w TSE). DWI was then acquired prior to injection of contrast agent in all cases using the following typical parameters for extracranial DWI: free-breathing transverse single-shot echo-planar imaging (SS-EPI-DWI), TR $=4600-$ $7544 \mathrm{~ms}, \mathrm{TE}=88-100 \mathrm{~ms}, \mathrm{~b}$-value $=50$ and $800 \mathrm{~s} / \mathrm{mm}^{2}, 5-10$ averages, voxel size: $1.2 \times 1.2 \mathrm{~mm}^{2}$ to $2.8 \times 2.8 \mathrm{~mm}^{2}$, slice thickness: 4-6 mm, acquisition time: $4-6 \mathrm{~min})$. Two scans for cranial osseous metastases were performed at b-values of 0 and $1000 \mathrm{~s} /$ $\mathrm{mm}^{2}$. The parameters FOV and number of slices were adapted as needed for optimal fit in each patient. ADC maps for the mean ADC (unit: $x 10-3 \mathrm{~mm}^{2} / \mathrm{s}$ ) were automatically calculated on the MRI console. After intravenous injection of one weight-adapted standard dose gadolinium-based contrast agent, fat-saturated sequences, e.g. T1w TSE for cervical and pelvic scans, T1w 2DFLASH (Magnetom Symphony) or CAIPIRINHA-accelerated 3DFLASH (Magnetom Aera) for thoracic-abdominal scans, were acquired as clinically appropriate with volume coverage and slice thickness corresponding to DWI. Patients aged $\leq 6$ years underwent MRI in sedation administered by a pediatric anesthesiologist.

\section{Image processing and analysis}

All image analysis was performed on a dedicated radiological workstation (Merlin Version 5.1., Phoenix PACS GmbH, Freiburg, Germany). A board-certified pediatric radiologist with 8 years of pediatric extracranial DWI experience (1st reader) performed complete readings of all examinations with full access to the routine radiological reports, histopathological reports and tumor board documents as reference. At this stage, tumor location, tumor size, presence/absence of metastases and tumor relapse and $A D C$ values of reference tissues (muscle tissue, liver, spleen renal cortex, pons cerebri, bulbus oculi, as included in the scan volume) were recorded. Two weeks later, the 1 st reader repeated the measurements of ADC values in reference tissues for the assessment of intra-observer variation and then measured ADC values of primary tumor manifestation, metastases and sites of tumor relapse using large polygonal regions of interest (large ROI) comprising the whole of the tumor on the level of its largest transverse cross-section, and small ROI with an area of 1 to $2 \mathrm{~cm}^{2}$, targeting tumor portions with the highest signal on DWI $b=800$ and corresponding low ADC, presumably representing those with the highest cellularity. Portions of the tumor showing a high DWI signal along with a high ADC were avoided, so as not to include T2 shine-through in our ADC data. Signal intensity of the tumor, muscle and liver tissue was measured with a small ROI on DWI $\mathrm{b}=800$ and on post-contrast T1w scans. The signal intensity of the surrounding tissue was measured in a similar fashion in a $1 \mathrm{~cm}$ perimeter of the tumor. All quantitative data were recorded as the mean value of three measurements. Signal intensity (SI) data was used to calculate signal intensity ratios (SIRs) dividing SI tumor by SI reference tissue as a measure of lesion conspicuity on DWI and on contrast-enhanced imaging.

For the analysis of inter-observer variability, a board-certified radiation oncologist with six years of experience in diagnostic MR imaging, MRI research and MRI-based radiation therapy eventually repeated the tumor-related quantitative measurements for all predefined lesions as a second reader, though not necessarily in the same slice position, while being blinded to data from the first reading. Prior to measuring study data, the two observers completed a non-blinded training session reading and analyzing ten data- 
sets of pediatric tumor patients not included in this study so as to arrive at a common strategy for measuring quantitative data.

\section{Statistical analysis}

Normally distributed data is presented as mean \pm standard deviation, and data deviating from normal distribution as median [interquartile range]. The Mann-Whitney test was used to compare means of unrelated samples, e. g. ADC of NB and GNB vs. ADC of GN. The Wilcoxon paired-sample test was employed to compare means of two related datasets, e. g. signal on DWI vs. signal on T1w imaging and ADC before and after therapy. P-values $<0.05$ were considered as indicating statistically significant differences. The coefficient of variation was computed to quantify intra-observer variation and inter-observer variation in quantitative data. All data analyses were performed with IBM SPSS Version 21 for Windows.

\section{Results}

\section{Neuroblastic tumors on initial imaging}

All tumor manifestations were identifiable and were visualized on both DWI and contrast-enhanced T1w imaging. The median tumor size was $46 \mathrm{~mm}$, ranging from 27 to $113 \mathrm{~mm}$, without a significant difference in size between DWI and ce-T1w imaging (paired sample t-test, $\mathrm{p}>0.05$ ). The mean ADC was $1.17 \pm 0.53$ [range: $0.61-2.33$ ] with a large ROI and $0.96 \pm 0.43$ [range $0.50-2.03$ ] with a small ROI for all tumors. NB/GNB had lower mean ADC values, compared to GN ( $\triangleright$ Table 1 ). A statistically significant difference was observed for NB vs. GN ( $p<0.001)$, for $N B+G N B(p<0.001)$ vs. $G N$ and for GNB vs. $G N(p=0.023)$. While mean $A D C$ was $\geq 1.18$ in all GN patients ( $\triangleright$ Fig. $2 A-C$ ), NB and GNB showed a consistently high signal on DWI $b=800$ with a correspondingly low mean $\mathrm{ADC} \leq 0.98$ ( $\triangleright$ Fig. 2E, F, 3A-C). A cut-off value of $\sim 1.3$ with large ROIs and $\sim 1.0$ for small ROIs grouped NB/ GNB vs. GN without overlap. The signal intensity ratios (SIR) of tumor vs. muscle and tumor vs. liver were significantly higher on DWI compared to ce-T1w ( $\triangleright$ Table 1$)$.

Comparing NB patients with $(n=4)$ and without $(n=4)$ MYCN gene amplification, MYCN-positive tumors showed a higher median tumor size $(10.2 \mathrm{~cm}$ vs. $3.6 \mathrm{~cm})$, lower median ADC with a large ROI (0.71 vs. 0.81$)$, lower median ADC with a small ROI (0.54 vs. 0.67 ) and lower median SIR tumor/surrounding tissue (5.3 vs. 6.6) compared to MYCN-negative lesions (all p >0.05).

\section{Therapy response in NB/GNB}

Imaging for therapy response was performed as scheduled by the referring pediatric oncologists, that is after 2 blocks of standard chemotherapy in 10 patients, after 3 blocks in 2 patients, after 1 block in 1 patient and after 6 blocks in 1 patient referred from a foreign institution. One patient showed extensive intralesional hemorrhage, as seen by partial intralesional signal loss on T2w and $\mathrm{T} 1 \mathrm{w}$ and by hyperintensity on native T1w, with artificially high signal at $b=800$ and consecutively false-low ADC ( $\triangleright$ Fig. 4) [16]. Eight of 15 patients had in-house pre-therapy scans. Com- paring tumor size on initial and on follow-up imaging in these patients, the median tumor size decreased from $53 \mathrm{~mm}$ to $23 \mathrm{~mm}$ post-therapy, while the mean ADC increased from $0.82 \pm 0.21$ to $1.72 \pm 0.33$ (large ROI) and from $0.63 \pm 0.14$ to $1.38 \pm 0.42$ (small ROI) (all $p<0.05$ ). The ADC of all post-therapy NB/GNB scans, excluding the one patient with intralesional hemorrhage, was $1.54 \pm 0.33$ with a large ROI and $1.21 \pm 0.41$ with a small ROI ( $\triangleright$ Table 1 ). The median diameter decreased by $56.6 \%$ (range: $1.5-75 \%$ ). A significantly higher SIR of tumor lesions was seen on DWI, compared to ce-T1w (all $p<0.01$ ), while the SIR on both DWI and on ce-T1w decreased with therapy $(p<0.05)(\triangleright$ Table 1$)$. $\Delta$ diameter showed a statistically non-significant correlation of $r=0.38(p=0.164)$ with mean ADC after therapy. The therapy-induced change in diameter was less than $25 \%$ in 2 of 15 patients with an $A D C \sim 1.3$ (large ROI) of the remaining tumor. One of these two patients died from tumor progression two months later in spite of continued therapy, while the other patient underwent further therapy with resection of liver metastases, I131-MIBI therapy, HD chemotherapy and autologous stem cell transplantation and is now in complete remission three years after primary diagnosis. One 18-month-old patient with cervical stage 3 NB showed spontaneous significant decrease in tumor size on physical examination and ultrasonography while preparing for chemotherapy and was put on "wait-and-watch". Follow-up MRI after 4 months showed a decrease in tumor diameter from 34 to $11 \mathrm{~mm}$ and an increase in ADC from 0.63 to 1.39 , and the tumor eventually resolved completely on further imaging follow-up.

\section{Therapy in GN patients}

Four of six GN patients underwent complete surgical resection. Subtotal resection was achieved in one patient with an adrenal $\mathrm{GN}$ and partial resection was performed in one patient with a large pelvic GN extending into the sacral foramina. The residual tumor remained stable on subsequent follow-up MRI imaging.

\section{Initial imaging and therapy response of synchronous metastases}

Sites of metastatic disease included cranial bone metastasis (patient 1), retroperitoneal lymph node and paravertebral intramuscular metastases (patient 2), retroperitoneal lymph node, multiple subcutaneous and pleural metastases (patient 3), as well as external iliac lymph node and pleural metastases (patient 4). Two metastatic manifestations in patient 3 and one distant external iliac lymph node metastasis in patient 4 were nearly occult on T2w and ce-T1w imaging, while clearly delineated on DWI. In total, four patients with eight metastatic lesions were analyzed. The initial median lesion diameter was $14 \mathrm{~mm}$ and the mean ADC was $0.67 \pm 0.14$ and $0.62 \pm 0.14$ with a large ROI and a small ROI, respectively ( $\vee$ Table 1 ). With therapy, four of eight lesions completely resolved without discernible residuals on DWI or ce-T1w. The remaining four lesions decreased in size by $29 \%$ to $80 \%$ and showed an increase in ADC to $1.31 \pm 0.24$ and 1.11 \pm 0.15 with a large ROI and a small ROI, respectively. There were two more metastasized patients without DWI on initial imaging. With therapy, retroperitoneal lymph node metastasis (patient 5) showed a $23 \%$ decrease in size in comparison to initial ce-T1w 
- Table 1 ADC values and signal intensity ratios (SIRs) of the various tumor entities on diffusion-weighted (DWI) and on contrast-enhanced (ce-T1w) imaging. ADC values (unit: $\left.x 10^{-3} \mathrm{~mm}^{2} / \mathrm{s}\right)$ are presented as mean \pm SD and range [minimum - maximum], the SIR values as median [inter-quartile range].

- Tab. 1 ADC und relative Signalintensitäten (SIR) der untersuchten Tumorentitäten mit Diffusions- (DWI) und kontrastverstärkter MRT (ce-T1w). Die ADC-Werte (Maßeinheit: x10-3 mm²/s) werden angegeben als Mittelwert \pm Standardabweichung und den Datenbereich [Minimum - Maximum], die SIR-Werte als Median [Interquartil-Abstand]

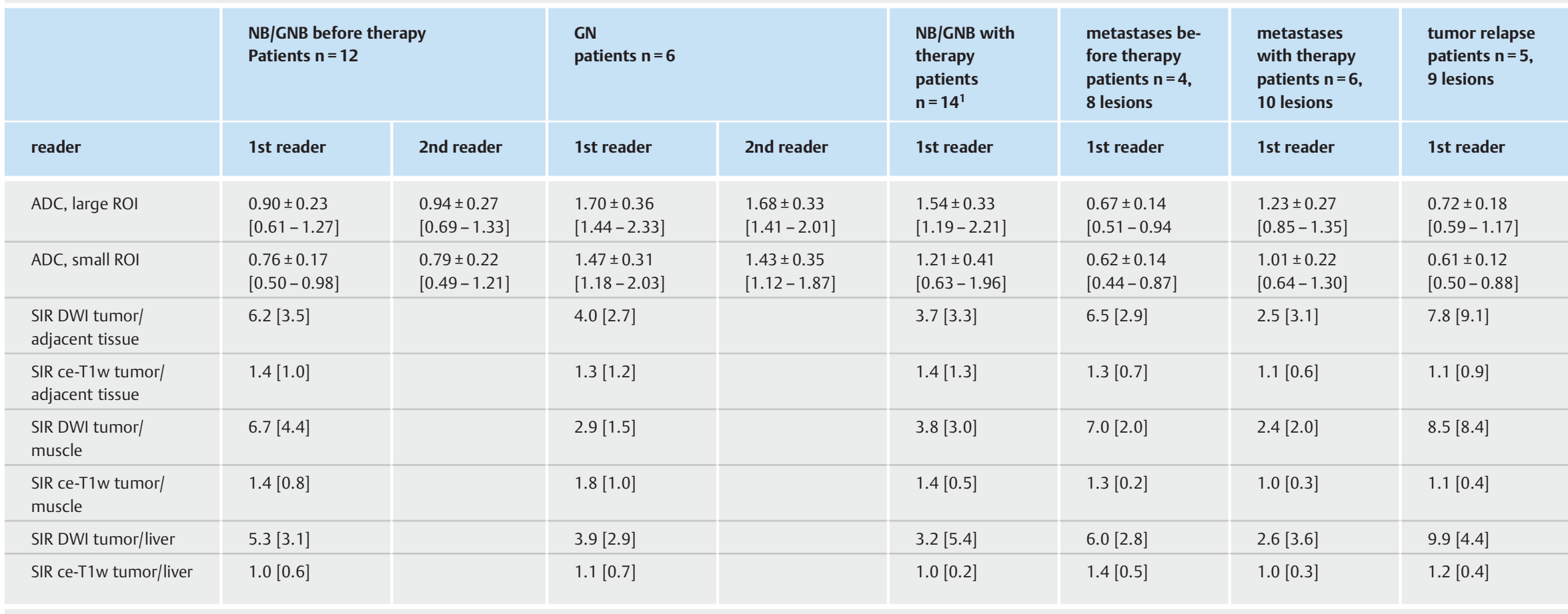

11 of $15 \mathrm{NB} / \mathrm{GNB}$ patients with therapy was excluded from quantitative analysis due to gross intralesional hemorrhage. 

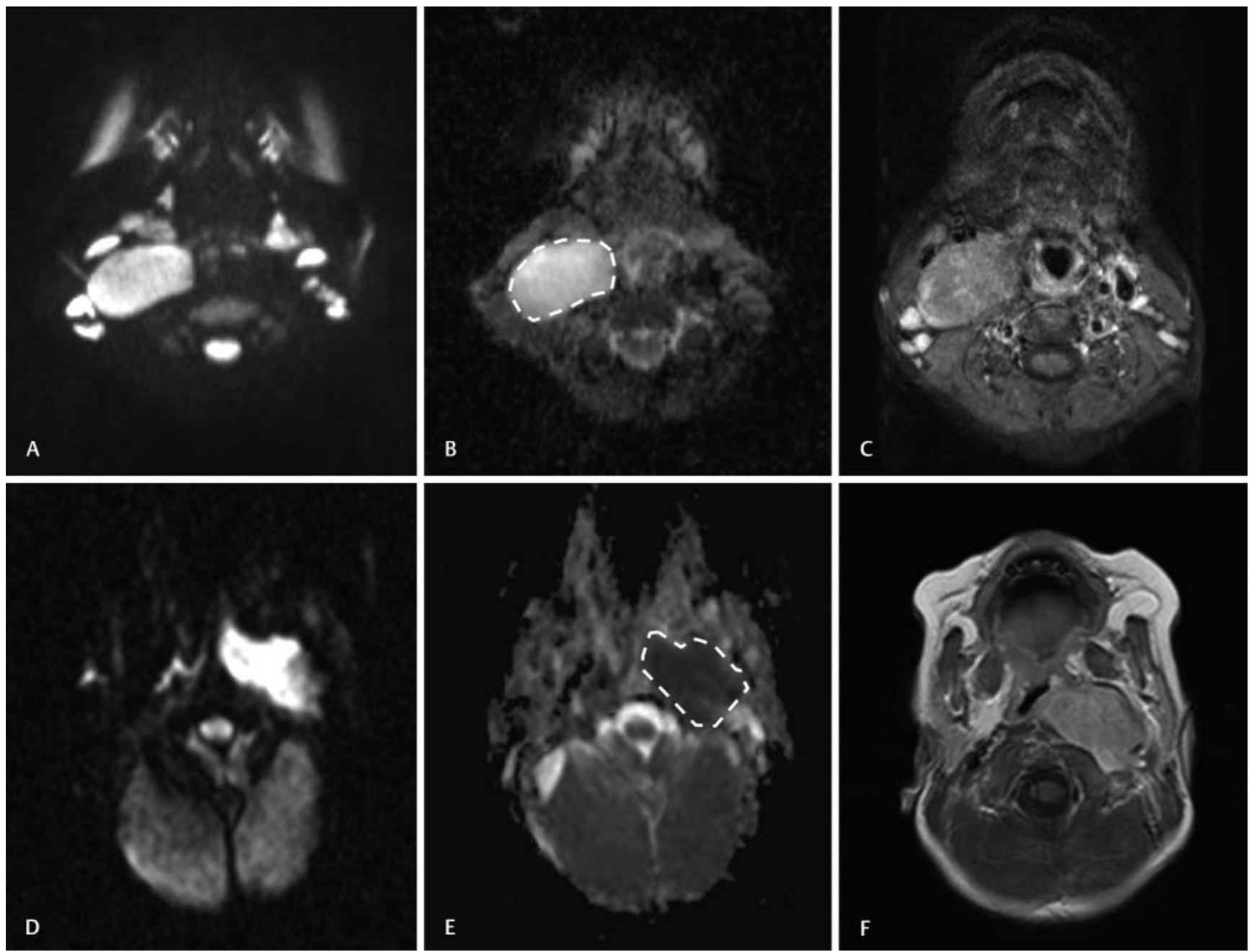

- Fig. 2 Seven-year-old boy with cervical ganglioneuroma A-C and 11-month-old boy with cervical neuroblastoma D-F. At initial imaging, both tumors are hyperintense on DWI b $=800 \mathrm{~A}, \mathbf{D}$ and show little contrast enhancement on ce-T1w TSE C, F. ADC was measured as 2.33 in GN B and 0.76 in NB E. The dotted line presents an exemplary large ROI. Examination A-C was performed on a 1.5 T Magnetom Aera scanner using traceweighted SS-EPI-DWI. Examination D-F was performed on an 1.5 T Magnetom Symphony with an orthogonal SS-EPI-DWI technique. No fat saturation was applied in $\mathbf{F}$.

- Abb. 2 7-jähriger Junge mit einem zervikalen Ganglioneurom A-C und ein 11 Monate alter Junge mit zervikalem Neuroblastom D-F. In der initialen Bildgebung sind beide Tumoren hyperintens im Diffusionbild mit b $=800$ A, D und zeigen nur geringe Kontrastmittelaufnahme in der T1 TSE C, F. Der mittels großer ROI gemessene ADC-Wert beträgt 2,33 für das GN B und 0,76 für das NB E. Die gepunktete Linie zeigt exemplarisch eine Messung mit großer ROI. Die Untersuchung A-C fand auf einem 1,5 T Magnetom Aera mit trace-weighted SS-EPI-DWI statt. Die Untersuchung D-F wurde auf einem 1,5 T Magnetom Symphony mit orthogonaler SS-EPI-DWI-Technik gemessen. Die T1w-Sequenz in $\mathbf{F}$ wurde nicht fettgesättigt.

imaging and an ADC with therapy of 1.29. Patient 6 with a large liver metastasis with a diameter of $60 \mathrm{~mm}$ on initial CT imaging was seen with a persisting liver metastasis of similar size $(63 \mathrm{~mm})$ and a low ADC of 0.85 and 0.64 measured with a large ROI and a small ROI, respectively, after three blocks of chemotherapy.

Analysis showed significantly higher SIRs of metastatic lesions on DWI, as compared to ce-T1w, both before and with therapy (all $p<0.01$ ). The SIR on both DWI and on ce-T1w decreased with therapy ( $\triangleright$ Table 1$)$.

\section{Imaging of tumor relapse}

Five patients in our study experienced one tumor relapse $(n=1)$ and repeated relapse/progression $(n=4)$. Tumor relapse involved the retroperitoneum in 3, the thoracic wall in 2 and bone metastases in 4 instances. The tumor ADC in patients with recurring lesions was low with $0.72 \pm 0.18$ (large ROI) and $0.61 \pm 0.12$ (small ROI). The SIR was very high on DWI ( SIR on contrast-enhanced imaging was similar to, or somewhat lower than, the SIR of initial tumor manifestations. Two patients underwent intensified or HD chemotherapy, which induced tumor response with a decrease in diameter by about $50 \%$ and an $A D C$ increase with a large ROI to 1.10 and 1.56 , respectively. One of these patients is presently still under treatment, the other four patients died from tumor progression. 

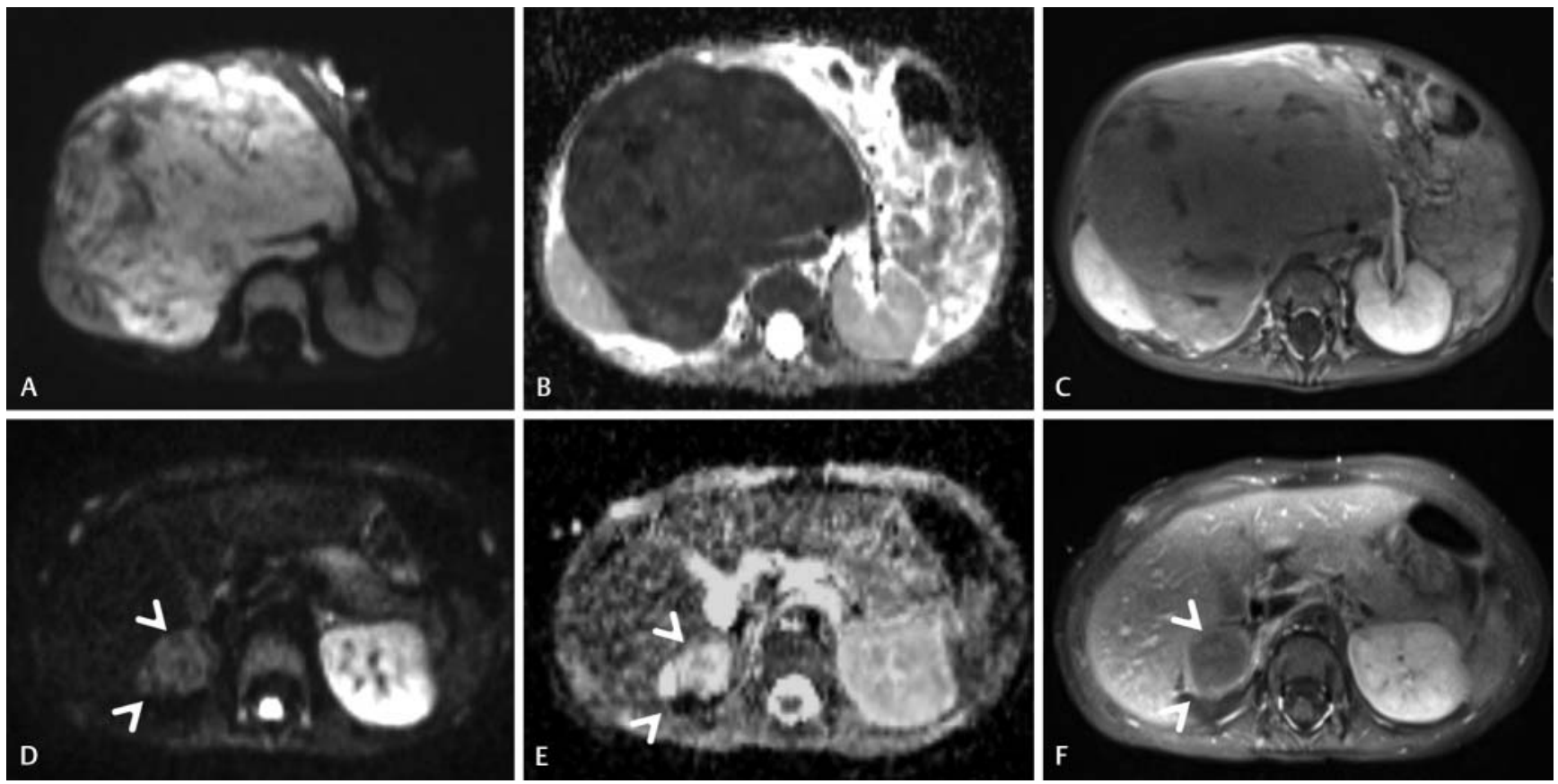

- Fig. 3 Imaging therapy response in a 2-year-old girl with abdominal neuroblastoma on initial imaging $\mathbf{A}-\mathbf{C}$ and after two blocks of standard chemotherapy D-F. The initially large and strongly hyperintense tumor on DWI b $=800$ A with low $A D C=0.61$ B shows little contrast enhancement on T1w TSE free-breathing $\mathbf{C}$. The right kidney is displaced and the large mesenteric vessels are encased by the tumor. There were paravertebral lymph node and intramuscular metastases (not shown). With treatment, the tumor diameter decreases by more than two-thirds, the remaining lesion shows a low DWI signal $\mathbf{D}$, high $A D C=2.2 \mathbf{E}$ and little, if any, contrast enhancement $\mathbf{F}$. The metastatic lesions resolved completely. Neither the primary tumor nor any of the metastatic lesions show significant FDG uptake on PET/CT performed within a few days of MR imaging before and after chemotherapy (not shown).

- Abb. 3 Therapieverlauf bei einem 2 Jahre alten Mädchen mit abdominellem Neuroblastom in der initialen Bildgebung A-C und nach 2 Blöcken Standard-Chemotherapie D-F. Der initial große und in der Diffusionsbildgebung mit b $=800$ A stark hyperintense Tumor mit niedrigem ADC $=0,61$ B zeigt kaum Kontrastmittelaufnahme in der freigeatmeten T1w TSE C. Der Tumor verlagert die rechte Niere und ummauert die großen mesenterialen Gefäße. Zusätzlich fanden sich paravertebrale Lymphknoten- und intramuskuläre Weichteilmetastasen (nicht im gezeigten Bild enthalten). Unter Therapie verringert sich der Tumordurchmesser um mehr als zwei Drittel. Der verbleibende Tumorrest zeigt nur geringe Hyperintensität im Diffusionsbild D, einen hohen $A D C=2,2$ E und kaum Kontrastaufnahme F. Die Metastasen waren unter Therapie nicht mehr abgrenzbar. Weder der Primärtumor noch die Metastasen zeigten eine wertbare FDG-Aufnahme im PET/CT (Bilder nicht gezeigt), das vor und nach Chemotherapie im Abstand von wenigen Tagen zur MRT durchgeführt wurde.

\section{Internal reference, intra-observer variability and inter-observer variability}

Mean ADC values of reference tissues were measured as follows: erector spinae muscle $1.15 \pm 0.10$, liver $1.06 \pm 0.20$, spleen 0.84 \pm 0.17 , renal cortex $1.61 \pm 0.17$, pons cerebri $0.82 \pm 0.06$, bulbus oculi $2.74 \pm 0.31$. No significant difference was found for ADC values comparing examinations performed on Magnetom Symphony and Magnetom Aera (all p >0.05).

The intra-observer coefficient of variation $\left(\mathrm{CV}_{\text {intra }}\right)$ was measured as follows: tumor ADC with a large ROI: $5.2 \%$, tumor ADC with a small ROI: $6.7 \%$, tumor signal at DWI $b=800: 7.1 \%$, muscle signal at DWI $b=800: 12.1 \%$, liver signal at DWI $b=800: 14.7 \%$, tumor signal at ce-T1w: 5.8\%, muscle signal at ce-T1w: $7.3 \%$, and liver signal at ce-T1w: $6.6 \%$. The $\mathrm{CV}_{\text {intra }}$ of the ADC in reference tissues was quantified as erector spinae muscle $6.8 \%$, liver $8.0 \%$, spleen $7.3 \%$, renal cortex $3.5 \%$, pons cerebri $6.8 \%$ and bulbus oculi $2.3 \%$.

An analysis of inter-observer variability showed the following inter-observer coefficients of variation $\left(\mathrm{CV}_{\text {inter }}\right)$ : tumor $A D C$ with a large ROI: $5.5 \%$, tumor ADC with a small ROI: $7.8 \%$, tumor signal at DWI b=800: $8.9 \%$, muscle signal at DWI $b=800: 13.8 \%$, liver signal at DWI b $=800: 16.4 \%$, tumor signal at ce-T1w: $6.2 \%$, muscle signal at ce-T1w: $8.7 \%$, and liver signal at ce-T1w: $7.5 \%$.

\section{Discussion}

Based on retrospective data from a large single-center patient cohort, our study results confirm the previously reported excellent diagnostic value of diffusion-weighted MRI for the initial diagnostic workup of pediatric patients with neuroblastic tumors. Ganglioneuroma, characterized by almost exclusively benign biological behavior, presented with distinctly higher mean ADC values than neuroblastoma and ganglioneuroblastoma. In NB/ GNB patients undergoing chemotherapy, DWI showed generally increasing $A D C$ values with some correlation between the degree of therapy response and the magnitude of ADC increase. Our study expands upon previously published work insofar that we demonstrate excellent lesion conspicuity for DWI of metastases 

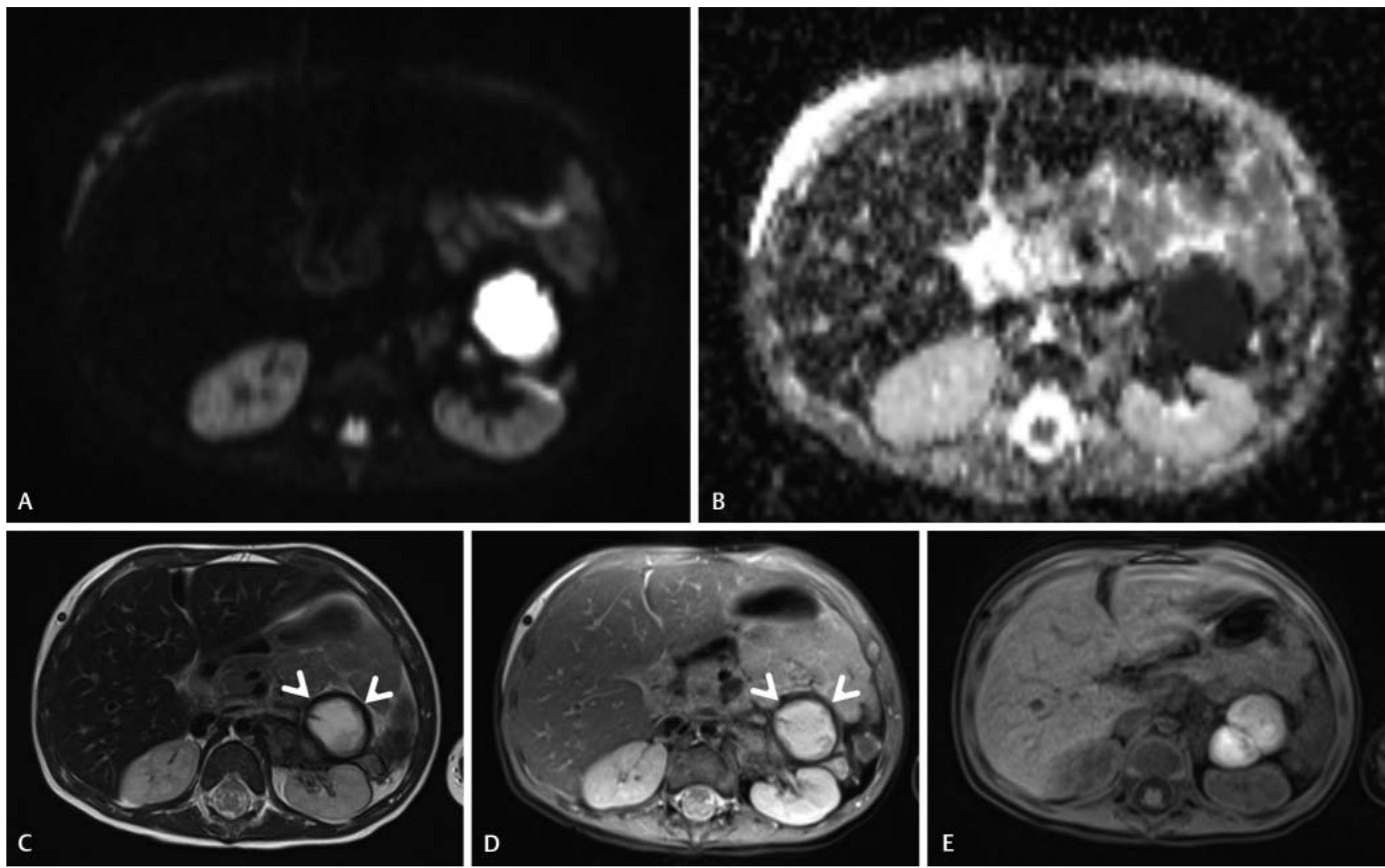

- Fig. 4 Diagnostic pitfall in an 18-month-old girl with abdominal neuroblastoma. The patient was referred from a foreign institution after 6 blocks of standard chemotherapy. Compared to initial CT imaging, tumor size had decreased from 75 to $41 \mathrm{~mm}$. On post-therapy MRI, the remaining tumor, however, was strongly hyperintense on DWI b $=800 \mathrm{~A}$ and had a very low $A D C=0.35$ B. Peripheral signal loss (arrowheads) on T2w TSE C and T1w TSE D suggested susceptibility artifacts due to intralesional hemorrhage, as supported by high intralesional signal on pre-contrast T1w 3DFLASH imaging E. Intralesional hemorrhage can cause complete signal loss on DWI [16], but also high signal with low corresponding ADC, thus mimicking restricted diffusivity and suggesting cell-rich tumor. The latter artifact is also a common finding with diffusion-weighted imaging of hemorrhagic renal cysts. As the MRI signal was not considered a reliable indicator of therapy response under these circumstances, we performed follow-up ultrasonography after three and after six weeks to verify continued shrinking and regression of the tumor.

- Abb. 4 Diagnostische Fallgrube bei einem 18 Monate alten Mädchen mit abdominellem Neuroblastom. Die Patientin wurde nach 6 Blöcken Standardchemotherapie von einer Klinik aus dem Ausland überwiesen. Im Vergleich zur initialen externen CT-Diagnostik hatte der Tumordurchmesser von 75 auf $41 \mathrm{~mm}$ abgenommen. Im MRT nach Therapie zeigte sich der Tumor jedoch stark hyperintens im Diffusionsbild $b=800 \mathrm{~A}$ und wies einen sehr niedrigen ADC-Wert von 0,35 auf B. In der Standardbildgebung T2 TSE und kontrastverstärkter T1 TSE wiesen der ringförmige periphere, am ehesten durch lokale Suszeptibilität bedingte Signalverlust im Tumor und ein Signalanhebung in der nativen T1 3D-FLASH E auf eine abgelaufene Einblutung hin. Tumoreinblutungen können zu einem kompletten Signalverlust in der DWI führen [16], aber auch mit Hyperintensität im Diffusionsbild und niedrigem ADC-Wert eine Diffusionsrestriktion, und damit zelldichten Tumor, vortäuschen. Das letztere Artefakt lässt sich häufiger in der Diffusionsbildgebung von eingebluteten Nierenzysten beobachten. Im Falle dieser Patientin konnte das Signalverhalten in der MRI nicht als zuverlässiger Parameter zur Beurteilung des Therapieverlaufs dienen, sodass das Therapieansprechen mittels sonographischer Kontrollen nach 3 und 6 Wochen anhand der fortschreitenden Größenreduktion des Tumors bestätigt wurde.

and tumor relapse. In addition, we provide quantitative data on lesion conspicuity comparing diffusion-weighted and contrastenhanced MRI. While metastases and relapsing tumor showed signal characteristics similar to primary NB before and after treatment, if only in a small sample of patients, lesion conspicuity with DWI was highly superior, as compared to standard MRI techniques.

The various entities of neuroblastic tumors in children cannot be distinguished reliably with standard MR imaging techniques $[4,5]$. The earliest report on restricted diffusivity in seven NB patients with a mean $A D C$ of 1.1 and a range of 0.9 to 1.2 dates back to 2002 [10]. In a study on children with a variety of malignant and benign tumors of the abdomen, three cases of neuro- blastoma imaged on a 1.5 T Magnetom Symphony scanner exhibited low mean ADC values of 0.70, 0.77 and 0.78 , while an $A D C$ cut-off value of 1.11 distinguished malignant and benign lesions with $100 \%$ sensitivity and $79 \%$ specificity [9]. Gahr et al. recorded a mean $A D C$ value of 0.81 , ranging from 0.39 to 1.47 , in $10 \mathrm{NB}$ patients, while $4 \mathrm{GN}$ and 2 GNB showed a mean ADC of 1.60, ranging from 1.13 to 1.99 [11]. A recent report on 24 children with $15 \mathrm{NB}, 5 \mathrm{GNB}$ and 4 GN observed a mean ADC of 0.869 in NB, 0.97 in GNB and 1.147 in GN with significant overlap and a proposed cut-off ADC value of 0.93 [12]. Our data on ADC of NB/ GNB and GN compare well to previously reported results. Depending on the ROI definition, all NB showed mean ADC values 

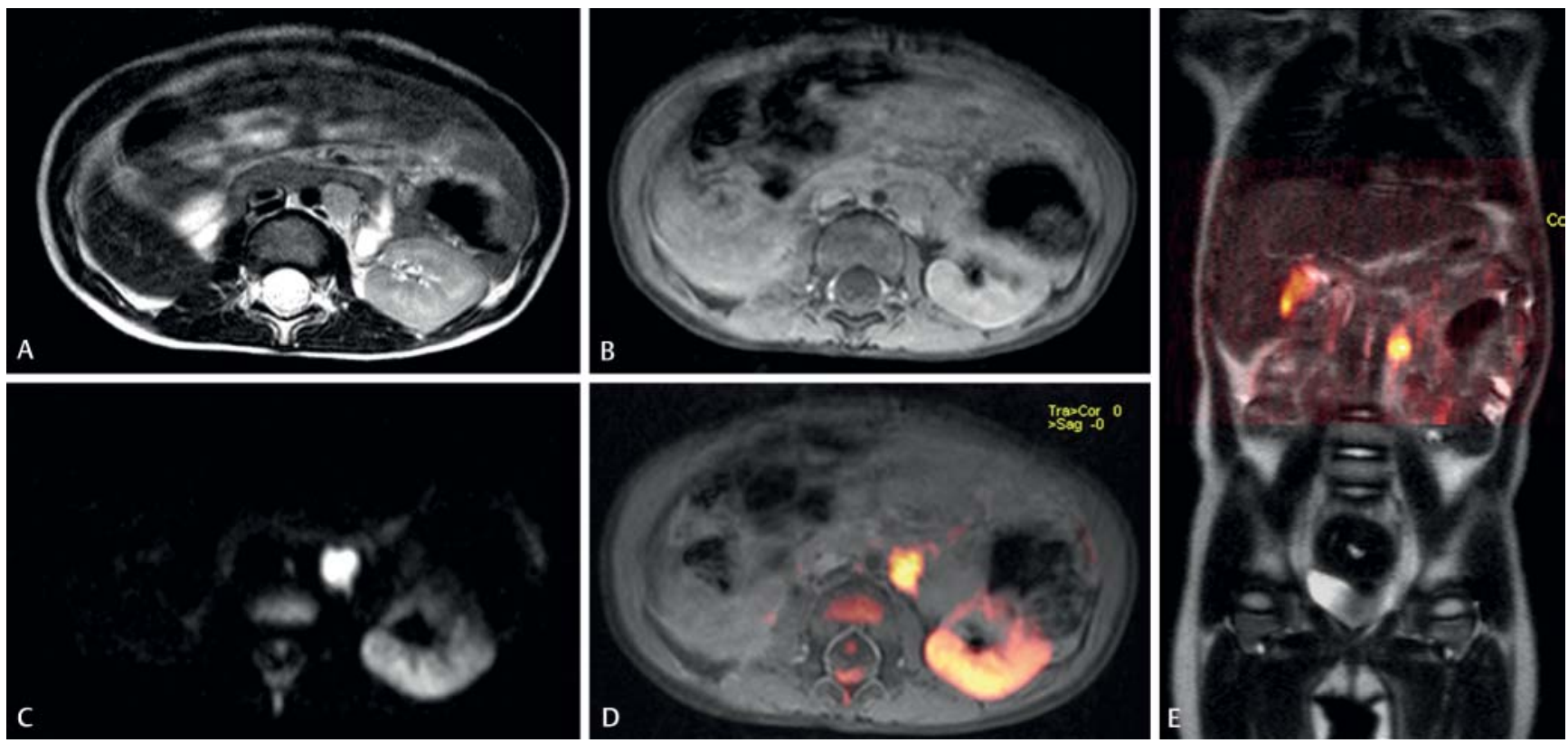

- Fig. 5 Paravertebral tumor relapse in a 30-month-old girl with abdominal neuroblastoma. The $14 \mathrm{~mm}$ lesion was difficult to detect on T2 TSE A and virtually occult on contrast-enhanced T1w imaging B. However, it clearly stood out on DWI b $=800 \mathrm{C}$ despite compromised overall image quality on the Magnetom Symphony scanner. The signal intensity ratio was 6.2 on DWI and 0.7 on ce-T1w for tumor vs. muscle, as well as 6.2 on DWI and 1.1 on ce-T1w for tumor vs. liver. Colorized overlay D of DWI onto the T1w image confirmed the location of the tumor lateral to the aorta and close to the vascular bundle of the left kidney. Image fusion of colorized DWI and T2w HASTE with coronal orientation $\mathbf{E}$ was found helpful for planning surgical resection.

- Abb. 5 Paravertebrales Tumorrezidiv bei einem 30 Monate alten Mädchen mit abdominellem Neuroblastom. Die 14 mm messende Läsion war in der T2 TSE A schwierig zu sehen und im kontrastverstärkten T1-Bild B nahezu okkult, in der Diffusionsbildgebung b=800 C jedoch anhand der fokalen Hyperintensität trotz ingesamt mäßiger Bildqualität am Magnetom Symphony nicht zu übersehen. Der Quotient der Signalintensitäten betrug 6,2 (DWI) und 0,7 (ce-T1w) für Tumor vs. Muskel sowie 6,2 (DWI) und 1,1 (ce-T1w) für Tumor vs. Leber. Die Bildfusion $\mathbf{D}$ von farbkodierter DWI mit dem T1-Bild bestätigte die Tumorlokalisation paraaortal am Gefäßstiel der linken Niere. Die multiplanare Darstellung des fusionierten DWI-Signals auf der coronaren T2 HASTE war für die Planung der Tumorresektion nützlich.

$\leq 1.27$ with a large $\mathrm{ROI}$, or $\leq 0.98$ with a small ROI. The ADC values we measured for GNB and GN were markedly higher than those reported by Serin et al. [12], resulting in a better discrimination between the tumor entities in our study. The underlying causes of this inconsistency remain unclear. Serin at al. also used Siemens hardware and free-breathing DWI. In their manuscript, however, they do not elaborate on scan parameters, such as TE and TR, single-shot vs. multi-shot acquisition, or indeed whether they used an SE sequence with EPI readout at all. Serin et al. scanned at b-values of 0 and 800 . The acquisition time given is rather short with $1 \mathrm{~min} 24 \mathrm{~s}$. As the manuscript does not provide any internal reference $A D C$, there is no objective approach to hypothesize whether the observable differences arise from a variation in technical parameters or rather from any of the numerous other potential intervening factors, such as different ethnicity, random variation in ADC with only four patients in the GN group, etc. We want to stress at this point, though, that we do not postulate a $100 \%$ discrimination of GNB from GN, or of malignant from non-malignant lesions, based on ADC values, in a larger patient cohort, or in the prospective setting of the clinical imaging routine. We do hold that measuring the mean ADC in neuroblastic tumors allows the best guess regarding biological malignancy that is presently possible based on data from noninvasive and radiation-free diagnostic procedures. Future research should address the potential correlation of tumor ADC with the presence or absence of MYCN gene amplification as a marker of high risk and poor outcome. Our data from very small subgroups showed a tendency towards a lower median ADC in MYCN-positive cases, a finding that warrants systematic investigation in a larger patient cohort.

In the present study, as well as in earlier reports, we observed a satisfyingly low degree of intra-observer and inter-observer variability for measured $A D C$ values with coefficients of variation usually less than $10 \%$, even across different DWI scanning techniques and on different scanners of the same manufacturer [17]. However, ADC variability arising from different acquisition techniques and from different scanner hardware needs to be taken into account. To illuminate this point, one patient with abdominal GN recently referred to our institution underwent initial MR imaging including DWI on a 1.5 T Intera scanner (Philips) at another imaging center. Exemplary quantitative analysis of this external dataset according to our study protocol showed the following results (reference data from our study in brackets): ADC of tumor with a large ROI: $1.13(1.70)$, ADC of tumor with a small ROI: 0.70 (1.47), placing the lesion well within the range of neuroblastoma, as defined by our in-house reference. However, this patient also showed a lower-than-usual ADC for reference tissues (reference data from our study in brackets): muscle 0.82 (1.15), liver 0.83 (1.06), spleen $0.55(0.84)$, renal cortex 1.09 (1.61). In order 
to facilitate between-study comparison, we therefore want to emphasize the usefulness of providing internal reference $A D C$ values from various body tissues.

Our findings confirm recent reports on the diagnostic value of DWI for monitoring therapy response in NB/GNB. Demir et al. [14] recorded significantly higher mean, maximum and minimum values for tumor ADC in 15 children undergoing DWI at 1.5 T (Magnetom Symphony and Magnetom Avanto) of 1.05 with therapy, compared to 0.74 on pre-therapy DWI, using a small ROI approach. These data are virtually identical to the data obtained with the small ROI evaluation in our study ( $\vee$ Table 1 ), further supporting our notion of good reproducibility of ADC quantification when using similar equipment and scanning parameters.

To the best of our knowledge, there are no previous reports on imaging characteristics of NB/GNB metastases and tumor relapse before and after chemotherapy. Although only a small number of such lesions were present in our study cohort, our preliminary findings suggest that these tumor manifestations exhibit imaging characteristics and signal changes on DWI very similar to those observed in primary tumors. Metastases and tumor relapse tend to have a lower mean ADC, compared to primary NB/GNB, perhaps reflecting an even higher malignant potency in these metastatic cell clones.

High signal intensity ratios for neuroblastic tumors on DWI perhaps present the most immediately useful finding from our study. Oncological MRI readings are expected to detect all tumor manifestations within a given scan volume. High intrinsic contrast of tumor lesions ensures lesion conspicuity and reliable visualization. Primary neuroblastic tumors are usually detected with diagnostic ease on any MR sequence due to their sizeable volume. Small metastatic lesions and early tumor relapse, on the other hand, are notoriously difficult to identify ( $\triangleright$ Fig. 5). Tumor lesions in our study demonstrated a generally low degree of hyperintensity on contrast-enhanced T1w imaging, as compared to the signal of muscle and liver tissue. Post-therapy metastases, tumor relapse, as well as untreated NB/GNB were nearly iso-intense to muscle and/or liver tissue on post-contrast MRI. In contrast, invariably high SIR values were seen on DWI, peaking at an 8.5to nearly 10 -fold signal intensity for tumor relapse vs. muscle or liver. These differences in SIR are very likely to translate into easier, faster and more reliable tumor detection with DWI. Contrast-tonoise ratio (CNR), widely used for measuring and comparing lesion conspicuity on MRI in the past, requires measured values on image noise and assumes homogeneous distribution of image noise. This precondition does not hold with multi-channel coils, various acceleration techniques and built-in image filtering which are now standard in modern scanner hardware. We therefore recommend and employ ratios of signal intensity as a quantifiable surrogate for lesion conspicuity.

Considering the findings from this study and from previous reports, we think that DWI should henceforth be considered an indispensable part of MR imaging protocols in patients with known or suspected neuroblastic tumor. The main limitation of this study, i. e. the relatively small patient cohort with incomplete sets of data examined on various MRI scanners, is hard to overcome at any given single imaging center. The absolute incidence of neuroblastic tumors in children is low and patient enrollment over several years is bound to witness changing imaging protocols with the arrival of new scanning techniques, or even new MRI hardware. When new scanner hardware was installed at our department in 2013, a period of transition was followed by the implementation of new scanning techniques, such as a switch from orthogonal SS-EPI DWI to trace-weighted acquisition and from standard contrast-enhanced FLASH imaging to Caipirinhaaccelerated FLASH scans. Each of the changes offered improvements in terms of image quality and faster image acquisition, however, also introduced another source of variation and potential bias into our study data. The in-house comparison of ADC values in reference tissues (muscle, renal cortex, liver etc.) across studies, scanners and acquisition protocols seems to indicate relative stability of ADC measurements with some variation which, in our view, is relatively small in contrast to other sources of variation, for instance, manual ROI segmentation. For the highly accelerated Caipirinha-FLASH scans, we demonstrated higher overall image quality [19] and equal-to-superior conspicuity of inflammatory bowel lesions, as compared to standard contrast-enhanced T1w imaging [20]. A study of tumor conspicuity comparing Caipirinha-FLASH and standard FLASH scans is still underway at our institution. Several of our patients were referred from other institutions after initial tumor staging on CT or MRI without DWI. In such patients, we did not repeat pre-therapeutic staging, so that data regarding pre-therapy imaging characteristics of primary tumor lesions were not available in these cases. Nevertheless, these patients were included in the analysis of post-therapy imaging studies. Eventually, as the number of metastatic lesions and relapse was low in our study cohort, we included up to two lesions per patient from different anatomic regions into the respective analyses, which is not an optimal approach from the statistician's point of view. Given the promising data available so far from clinical studies, including this study, study centers should become more aware of the high potential of diffusionweighted scanning and promote the use of DWI in standardized imaging protocols. In a multicenter setting, the diagnostic performance of DWI for tumor detection and for tumor differentiation should prospectively be tested against contrast-enhanced MRI to see whether the preliminary findings published so far regarding neuroblastic tumors hold for a larger, prospectively enrolled patient cohort and whether the application of i. v. contrast agent actually adds diagnostic value to an MRI examination comprising native T1w, T2w and diffusion-weighted imaging. MRI with DWI should also prospectively be evaluated in comparison to diagnostic MIBG scan and PET/CT scans. PET/CT has recently been presented as providing additional prognostic information in NB patients [18]. However, NB cases without FDG uptake are not infrequent, as in our patient presented in Fig. 3. Similarly, only $90 \%$ of NB [21] and only $50 \%$ of NB relapses [22] show MIBG uptake. Studies on NB patients reported high cumulative [23] and per-examination [24] doses of radiation exposure from $\mathrm{PET} / \mathrm{CT}$. Excessively high cumulative doses were reported from $\mathrm{PET} / \mathrm{CT}$ scans ranging up to $399 \mathrm{mSv}$ per patient in a study on children with malignancies [25], thus counteracting all efforts for dose reduction in pediatric patients achieved in recent years by increased usage of MR imaging. Many children with neuroblastic tumors enjoy an excellent long-term prognosis and should not 
be exposed to high radiation doses without need. While PET/MRI may be one approach to reduce radiation exposure from wholebody scans in the future, whole-body DWI as a radiation-free, cost-effective and readily available alternative should be evaluated prospectively head-to-head with PET/CT, PET/MRI and diagnostic MIBI scans [13]. Finally, concerns have recently been raised about the long-term safety of gadolinium-based contrast agents, based on the observation of intracranial gadolinium deposition in patients after contrast-enhanced MRI scans [26]. Based on our preliminary experience, DWI may serve as a useful diagnostic tool to perform native MRI scans with high intrinsic tissue contrast and to substitute at least some Gd-enhanced MRI scans. However, more data and higher evidence seems necessary in this respect in order to support more definite conclusions.

\section{CLINICAL RELEVANCE OF THE STUDY}

- Our study results provide evidence that diffusion-weighted MRI as a noninvasive, radiation-free, cost-effective, fast and high-contrast imaging tool provides reproducible quantitative parameters for comprehensive oncological imaging in pediatric patients with neuroblastic tumors.

- DWI is available on all modern MRI scanners and is to be recommended as part of standard MRI scanning protocols for childhood cancer.

- Whole-body DWI should undergo prospective evaluation in a head-to-head comparison with contrast-enhanced MRI, PET/CT and MIBI scans.

\section{Conflict of Interest}

The authors declare that they have no conflict of interest.

\section{References}

[1] Joshi VV. Peripheral neuroblastic tumors: pathologic classification based on recommendations of international neuroblastoma pathology committee (modification of Shimada classification). Pediatr Dev Pathol 2000; 3: $184-199$

[2] Spix C, Aareleid T, Stiller C et al. Survival of children with neuroblastoma. Time trends and regional differences in Europe, 1978-1992. Eur J Cancer 2001; 37: $722-729$

[3] Maris JM. Recent advances in neuroblastoma. N Engl J Med 2010; 362: $2202-2211$

[4] Lonergan G], Schwab CM, Suarez ES et al. Neuroblastoma, ganglioneuroblastoma, and ganglioneuroma: radiologic-pathologic correlation. Radiographics 2002; 22: 911 - 934

[5] McCarville MB. What MRI can tell us about neurogenic tumors and rhabdomyosarcoma. Pediatr Radiol 2016; 46: 881 - 890

[6] Brisse HJ, McCarville MB, Granata C et al. Guidelines for imaging and staging of neuroblastic tumors: consensus report from the International Neuroblastoma Risk Group Project. Radiology 2011; 261: 243 -257

[7] Ley S, Ley-Zaporozhan J, Günther P et al. Neuroblastoma imaging. Roefo 2011; 183: 217-225
[8] Pinto NR, Applebaum MA, Volchenboum SL et al. Advances in Risk Classification and Treatment Strategies for Neuroblastoma. J Clin Oncol 2015; 33: 3008-3017

[9] Kocaoglu M, Bulakbasi N, Sanal HT et al. Pediatric abdominal masses: diagnostic accuracy of diffusion weighted MRI. Magn Reson Imaging 2010; 28: 629-636

[10] Uhl M, Altehoefer C, Kontny U et al. MRI-diffusion imaging of neuroblastomas: first results and correlation to histology. Eur Radiol 2002; 12: $2335-2338$

[11] Gahr N, Darge K, Hahn G et al. Diffusion-weighted MRI for differentiation of neuroblastoma and ganglioneuroblastoma/ganglioneuroma. Eur J Radiol 2011; 79: $443-446$

[12] Serin HI, Gorkem SB, Doganay S et al. Diffusion weighted imaging in differentiating malignant and benign neuroblastic tumors. Jpn J Radiol 2016; 34: 620-624

[13] Pai Panandiker AS, Coleman J, Shulkin B. Whole Body Pediatric Neuroblastoma Imaging: 123I-mIBG and Beyond. Clin Nucl Med 2015; 40: $737-739$

[14] Demir S, Altinkaya N, Kocer NE. Variations in apparent diffusion coefficient values following chemotherapy in pediatric neuroblastoma. Diagn Interv Radiol 2015; 21: 184-188

[15] Brodeur GM, Pritchard J, Berthold F et al. Revisions of the international criteria for neuroblastoma diagnosis, staging, and response to treatment. J Clin Oncol 1993; 11: 1466-1477

[16] Sauer A, Müller V, Pabst T et al. Diffusion-weighted MRI for diagnosis and differential diagnosis of lymphatic malformation in paediatric patients. Cancer Research Frontiers 2016; 2: $300-310$

[17] Neubauer H, Evangelista L, Hassold $\mathrm{N}$ et al. Musculoskeletal tumorous and tumour-like lesions: diagnostic utility of diffusion-weighted MRI for detection and differentiation in pediatric patients. World J Pediatr 2012; 8: $342-349$

[18] Lee JW, Cho A, Yun M et al. Prognostic value of pretreatment FDG PET in pediatric neuroblastoma. Eur J Radiol 2015; 84: 2633-2639

[19] Li M, Winkler B, Pabst T et al. Fast MR imaging of the paediatric abdomen with CAIPIRINHA-accelerated T1w 3D-FLASH and with high-resolution T2W HASTE: a study on image quality. Gastroenterol Res Pract 2015. DOI: $10.1155 / 2015 / 693654$

[20] Li M, Dick A, Hassold N et al. CAIPIRINHA-accelerated T1w 3D-FLASH for small-bowel MR imaging in paediatric patients with Crohn disease: assessment of image quality and diagnostic performance. World J Pediatr 2016; 12: 455-462

[21] Sharp SE, Trout AT, Weiss BD et al. MIBG in Neuroblastoma Diagnostic Imaging and Therapy. Radiographics 2016; 36: 258-278

[22] Andrich MP, Shalaby-Rana E, Movassaghi N et al. The role of 131 iodinemetaiodobenzylguanidine scanning in the correlative imaging of patients with neuroblastoma. Pediatrics 1996; 97: 246-250

[23] Kim YY, Shin H], Kim M] et al. Comparison of effective radiation doses from X-ray, CT, and PET/CT in pediatric patients with neuroblastoma using a dose monitoring program. Diagn Interv Radiol 2016; 22: 390 394

[24] Federman N, Feig SA. PET/CT in evaluating pediatric malignancies: a clinician's perspective. J Nucl Med 2007; 48: 1920-1922

[25] Chawla SC, Federman N, Zhang D et al. Estimated cumulative radiation dose from PET/CT in children with malignancies: a 5-year retrospective review. Pediatr Radiol 2010; 40: 681-686

[26] Stojanov D, Aracki-Trenkic A, Benedeto-Stojanov D. Gadolinium deposition within the dentate nucleus and globus pallidus after repeated administrations of gadolinium-based contrast agents - current status. Neuroradiology 2016; 58: 433-441 